\title{
Design of a Monitoring System for the Measurement of Surrounding Rock Deformation in Underground Tunnels
}

\author{
Zhang Hongqing, Fan Zhiqiang, Chen Qing, Lu Ke, Wang Chunyan
}

Northwest Institute of Nuclear Technology, Xi'an 710024, China

Keywords: Deformation Monitoring; Non-contact Measurement; Unattended Operation; Low-power Consumption

\begin{abstract}
This study designed an automatic monitoring system for non-contact measurement of the surround rock deformation in underground tunnels in an unattended operation mode. This system can automatically and periodically monitor the deformation of the surrounding rocks in underground tunnels with low power consumption, and write the monitored deformation data to the TF card in the format of .txt. Accordingly, the surveyors need only get the monitoring data from the TF card regularly and analyze the surrounding rocks'deformation trend.
\end{abstract}

\section{Introduction}

Underground tunneling can destroy the original stress balance on the surrounding rocks, leading to deformation and failure. Therefore, the quantitative assessment of the deformation of surrounding rocks in underground tunnel is quite necessary for optimizing support parameters, achieving in-time warning and ensuring the safety in tunneling construction.

Currently, deformation monitoring tasks for the surrounding rocks in underground tunnel rely mainly on everyday manual measurement, i.e., the engineering surveyors enter the monitoring sections in the tunnel at a fixed time every day and conducted the measurements using rulers or total-station instrument. Accordingly, the surveyors should pull the rule or install the total-station instrument on the site regularly, causing heavy workload. Moreover, the project would be delayed to certain degree; in particular, at some unapproachable positions, the surveyors would have to get access to the dangerous regions for acquiring data, which would pose a threat to their safety. To overcome these drawbacks in manual operation, this study designed a low-cost field deformation monitoring system for the surroundingrocks in underground- projects.

\section{A brief introduction of the system}

Based on laser ranging, the designed system can operate underground for a long time for conducting periodic distance measurement on the mark points that were set on the surrounding rock walls in advance (as shown in Fig. 1). Moreover, the measured data as well as measurement time were automatically saved in the T-flash (TF) card in the system. The whole system was powered by the dry battery. Assumed that the measurement was conducted every two hours, three AAA batteries can supply the power for approximately a month. During the measurements, the surveyors can copy the data from the measurementsystem at any time for gaining real-time data of the deformation of the surrounding rocks.

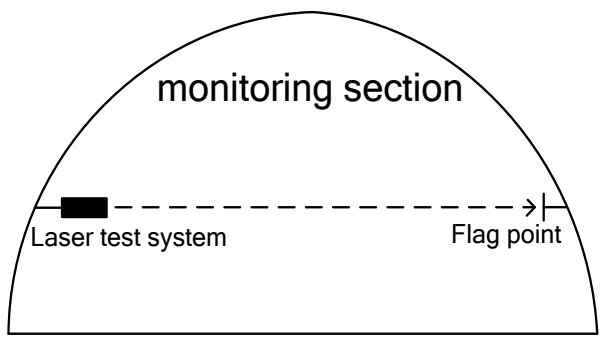

Fig.1 Principle of the monitoring system 


\section{Key technologies in the system}

\subsection{Design of operating mode}

The system hardware was developed based on the STM32F103 microprogrammed control unit (MCU) provided by ST Microelectronics. For minimizing the system consumption, we should simplify the hardware design and reduce system overhead, but the key lies in the design of system operating mode.

After startup, the system automatically enters into unattended operation mode, i.e., the system is in sleep mode before measurement for reducing the battery capacity consumption; when the timing module in the system reaches the preset wake-up time, the system is automatically switched on for laser ranging, and the measured data and the corresponding measurement times are saved to the T-flash (TF) card in the control circuit in the format of .txt; then, the system enters into the sleep mode again.

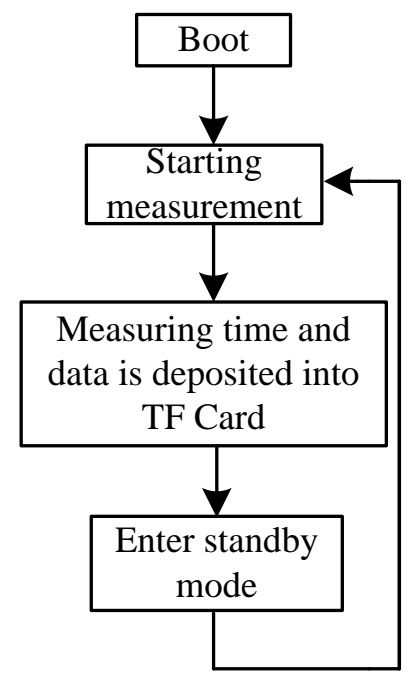

Fig.2 Operating mode of system

\subsection{Power design}

\subsubsection{Power-switching circuit}

During the operation, the system was powered by three dry batteries in series (each with a direct voltage of $1.5 \mathrm{~V}$ and an output in total of $4.5 \mathrm{~V}$ ); via a low-consumption power switching chip (LM3670, with a quiescent current of uA order), a $3.3 \mathrm{~V}$ current voltage was output and supplied to MCU and the laser ranging sensor for maintaining their normal operation.

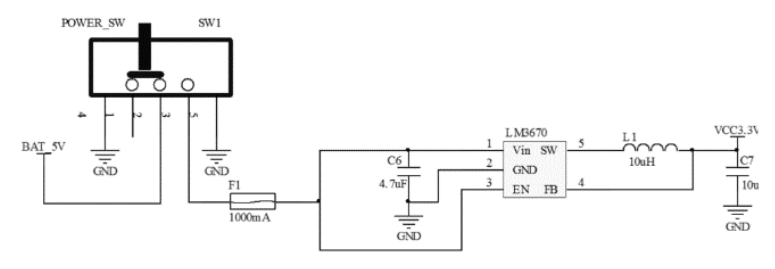

Fig.3 Supply circuit of the system

Supply circuit

\subsubsection{Switching circuit for the laser sensor}

The used laser sensor still consumed an approximately $30 \mathrm{~mA}$ standby current in standby mode, which seriously shortened the battery life. For completely cutting off the sensor's consumption power in standby state, a switching on-off circuit was designed, as shown in Fig. 4, in which P-type MOS tube (SI1317DL) was used. 


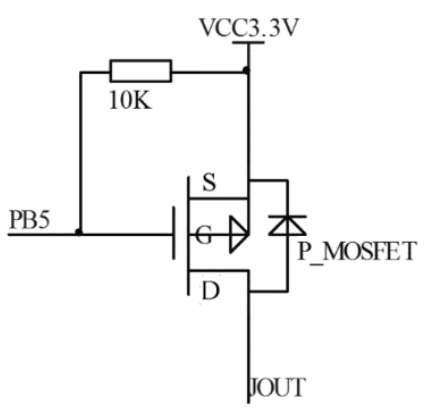

Fig. 4 Switching circuit for the sensor

The laser sensor was controlled by high/low level output from the PB5 pin of the MCU. Specifically, high level turned off the circuit while low-level turned on the circuit. A $10 \mathrm{k}$ pull-up resistor was set for ensuring that the MCU can be shut off in reset and failure modes.

\subsubsection{Power for timing module}

Built-in real time clock (RTC) in the MCU was adopted as the system timing module. Since RTC operated in the MCU's backup area, the backup area can still be powered by an external $3.3 \mathrm{~V}$ button cell (as shown in Fig. 5) when the external power supply was cut off, and time record was not changed by external power interruption, so that the time accuracy in the record can be guaranteed.

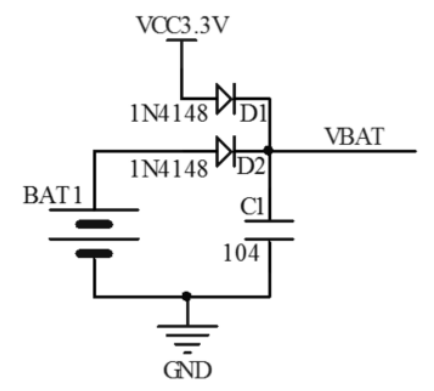

Fig. 5 Supply circuit for RTC

\subsection{Data storage}

In designing the software of MCU, FAT32 file system was migrated, and then, the laser ranging data were directly written in TF card in the format of .txt via SPI port. The surveyors can directly take out TF card in field operation and view the data on their carry-on notebook computer.

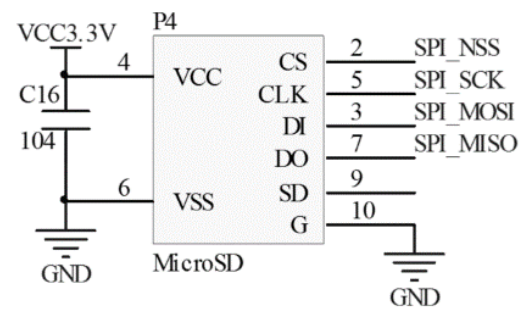

Fig.6 Circuit of SPIport

\section{System testing}

\subsection{Deployment of the system}

The tests were carried out in an underground tunnel under construction. According to the requirements in field test, the measuring system was deployed, as shown in Fig. 7. A measurement system was arranged on each section.

Aluminum shield was used for protecting the measurement system from the pollution. The mounting holes were reserved on the side wing of the shield. In field operation, the system was 
directly installed to the mounting plate, while the anchored rebar was casted into the rock wall, with an insertion depth of approximately 40 50 m.

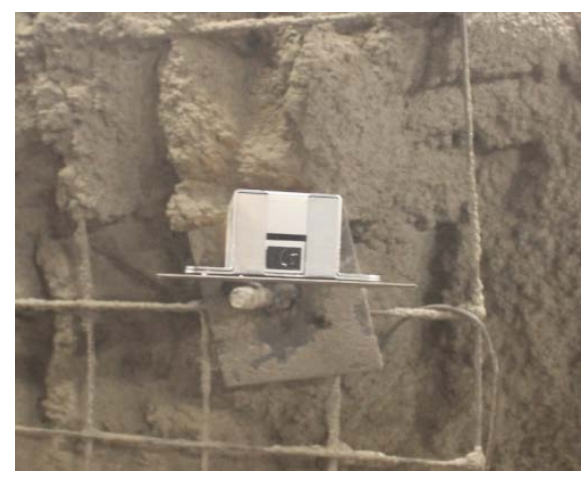

Fig. 7 Layout of the measurement system

Laser-based ranging generally uses pulse laser and derives the distance data according to the diffusion reflection signal of the laser by the target object. Therefore, ranging results are tightly related to the reflection effects of the mark point. The laser market point should be set on the rock wall opposite to the measurement system for the reflection of detecting laser (as stated above, the anchored rebar was casted into the rock wall by approximately $40 \sim 50 \mathrm{~m}$ ). Fig. 8 displays the stainless steel mark point on the reflecting surface.

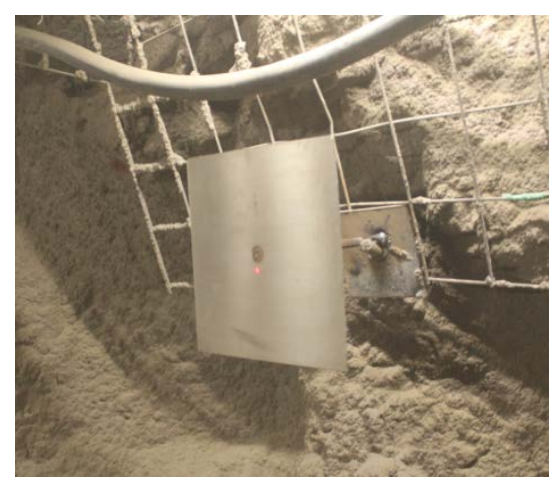

Fig. 8 Layout of the laser mark point

\subsection{Standard deviation}

Next, the laser sensing point aimed at the rock wall at approximately $5 \mathrm{~m}$ away from the measurement system, and the system was set to be wakened every hour. The system was powered on at 18:02, and 10 measurements in total were conducted, as the monitoring data listed in Table 1.

Table 1 Monitoring data by the measurement system (unit: $\mathrm{m}$ )

\begin{tabular}{|l|c|c|c|c|c|}
\hline Test No. & 1 & 2 & 3 & 4 & 5 \\
\hline Measurement data & 5.955 & 5.955 & 5.956 & 5.955 & 5.955 \\
\hline Test No. & 6 & 7 & 8 & 9 & 10 \\
\hline Number of tests & 5.955 & 5.955 & 5.955 & 5.956 & 5.955 \\
\hline Standard deviation & \multicolumn{5}{|c}{0.00042} \\
\hline
\end{tabular}

\section{Conclusions}

Based on laser ranging technique, this study designed a non-contact measurement system for monitoring the deformation of the surround rocks in underground tunnels. This system can achieve long-time stable operation in underground tunnels in the absence of external power supply; meanwhile, the system's operation only needs the pre-deployment of measuring pointwithout 
subsequent manual maintenance. Owing to the built-in of TF card, the cost of long-distance layout of measuring cables as well as he operating cost was greatly reduced, data acquisition process was simplified. In future work, we attempt to add wireless transmitting and receiving module for further facilitating data acquisition.

\section{References}

[1] Wang Mengshu. Tunneling and Underground Engineering Techno -logy in China [M]. Beijing: China Communication Press, 2010. 5.

[2] Qing Haitao. Design of the Wireless Deformation Monitoring System with Micro-power Consumption for Roadway Wall Rock [J].Process Automation Insturmentation, 2014, 35(11).

[3] Zhu Wenjie, HaoJingbin, Yang Q L, et al..Laser Measurement Method for Surface Deformation of Roadway Surrounding Rock [J]. Safety in Coal Mines, 2015, 46(6).

[4] Liu Huoliang and Yang Sen. STM32Base Development Manual [M]. Beijing: China Machine Press, 2013.6.

[5] Chinese Version of STM32 Reference Manual [M]. ST Official Website, 2009.12. 